\title{
When Can AI Reduce Individuals' Anchoring Bias and Enhance Decision Accuracy? Evidence from Multiple Longitudinal Experiments
}

\author{
Kyootai Lee \\ Graduate School of Management of Technology \\ Sogang University \\ kyootai@sogang.ac.kr \\ Wooje Cho \\ School of Business \\ Seoul National University \\ woojecho@snu.ac.kr
}

\author{
Han-gyun Woo \\ School of Business Administration \\ UNIST \\ hwoo@unist.ac.kr \\ Simon B. de Jong \\ School of Business and Economics \\ Maastricht University \\ s.dejong@maastrichtuniversity.nl
}

\begin{abstract}
This study aimed to identify and explain the mechanism underlying decision-making behaviors adaptive to AI advice. We develop a new theoretical framework by drawing on the anchoring effect and the literature on experiential learning. We focus on two factors: (1) the difference between individuals' initial estimates and AI advice and (2) the existence of a second anchor (i.e., previous-year credit scores). We conducted two longitudinal experiments in the corporate credit rating context, where correct answers exist stochastically. We found that individuals exhibit some paradoxical behaviors. With greater differences and no second anchor, individuals are more likely to make adjustment efforts, but their initial estimates remain strong anchors. Yet, in multiple-anchor contexts individuals tend to diminish dependence on their initial estimates. We also found that the accuracy of individuals was dependent on their debiasing efforts.
\end{abstract}

\section{Introduction}

Researchers [e.g., 1, 2] have highlighted that AI can accentuate biases in human decision making and that decision makers must attempt to understand the fairness of algorithms. Additionally, existing literature demonstrates that individuals can hold the contradictory biased attitudes of algorithm appreciationindividuals' adherence to algorithmic advice compared to human advice [3] - and algorithm aversionindividuals' aversion to algorithmic recommendations [4, 5]. Researchers have identified several factors characterizing decision-making contexts that could mitigate algorithm aversion (or appreciation), such as expertise [3] and the power to create alternatives [5]. This means that different individuals may interpret the value of AI advice differently. Such contradictory attitudes may depend on how individuals perceive the value vis-à-vis their preexisting judgements in decisionmaking processes and how AI advice is provided. Additionally, regarding the evolutionary nature of individuals' attitudes, researchers [6] have highlighted dynamic aspects such as changes in users' attitudes toward new information technologies while experiencing them as part of their decision making. Thus, individuals' algorithm aversion and appreciation may evolve depending on their experiences in AI-aided decision-making environments (e.g., initial algorithm aversion then algorithm appreciation). Because AI possesses a distinct characteristic-reinforcement learning [1] - forms of AI adopted by individuals should be considered as dynamic rather than static, potentially changing individuals' attitudes and behaviors. A key issue is that existing theories do not adequately address individuals' attitudes and behaviors in explaining why and how they adapt (or do not adapt) in AI-aided decision-making contexts over time. To our knowledge, current research provides only limited theoretical insights about the individuals' adaptation [6] and limited perceptual evidence on how the technology may influence individuals' performance over time.

Consider an experienced manager asked to estimate the asset value of a company. Although the manager does not know the true value, they have an initial estimate (a self-generated first anchor) of \$300 million based on their own analysis of the information, their heuristics, and their expertise. The manager can also refer to a value from the previous year (an externally generated second anchor), which could be $\$ 295$ million, for example. When AI suggests a valuation of $\$ 320$ million, then the manager might update their estimate to \$315 million. If the company later sells for \$316 million, the manager's belief in the AI recommendations may increase, which may reinforce the manager's attitudes toward AI and thus increase reliance on its recommendations in future decision making. However, if the company sells for $\$ 305$ million, the opposite may 
occur. This example implies that individuals' attitudes toward AI advice may be not only a function of their own decision-making strategies, with respect to their susceptibility to anchoring biases, but also a function of outcomes from their experiences using AI.

While addressing existing studies' limitations, this study explores the following issues that can influence individuals' attitudes toward AI advice. First, decision makers may perceive that AI advice significantly deviates from their own initial estimates, which can act as self-generated anchors. In this case, it is worth asking whether individuals exhibit the anchoring biasrepresenting decision makers' tendency to consider irrelevant information [7] — in the context of AI-aided decision making [8]. Second, individuals within organizations tend to receive information from various sources, some of which can be inconsistent. Expanding the first issue to multiple anchors [9], how do individuals consider AI advice, which may or may not be consistent with that of other information sources? One possibility is that if multiple anchors are available to individuals [9], this may mitigate the influence of AI advice on individuals' final decisions. Third, AI often needs to learn from decision makers' expertise and heuristics when correct answers do not exist a priori and irreducible uncertainties are prevalent. Individuals also learn the qualities of AI while adapting to AI-aided decision-making contexts. Considering such AI-user coevolution, do AI and its users assimilate to each other? If so, perhaps AI can help individuals overcome the anchoring bias. Finally, can AI help individuals make decisions more accurately? Though individuals have the tendency to rely on heuristics to make judgments [10], such tendencies may lead to cognitive biases that can have detrimental effects. Hence, this study focuses on determining whether and how AI advice can help enhance individuals' decision accuracy.

We conducted two longitudinal experiments in the corporate credit rating context, in which correct answers exist stochastically, akin to many other non-routine tasks in organizations. Therefore, AI can help users make decisions in uncertain contexts by providing statistically better (but not correct) predictions [1]. In the first experiment, we examined the effect of the difference between individuals' initial estimate and AI advice on their decision-making behaviors. In the second experiment, we expanded the first experiment to multiple anchor contexts by providing a second anchor (previous-year credit score). While differentiating the conditions, we consistently consider individuals' experiential learning in the two experiments as individuals were asked to rate credits for ten companies

\footnotetext{
${ }^{1}$ The accuracy of AI was higher than that of its users in our experiments. Detailed results are provided in the method sections.
}

per session. By doing so, we could better identify how AI technologies influence choice shifts and, furthermore, how AI becomes an integrated part of human decision making via interactions with users [6].

\section{Hypotheses}

We employ experiential learning theory [11] and anchoring effects $[8,12-14]$ to understand individuals' attitudes toward AI advice and the advice's effects on their final choices over time. We follow Simmons et al. [15] integrative theory of anchoring, combining the anchoring-and-adjustment model and selective accessibility model to better understand how individuals accept AI advice and the extent to which they adjust their own judgments after receiving the advice. According to Simmons et al, individuals' debiasing behaviors are likely to depend on their confidence about the direction of their initial choice shifts. Additionally, we adopt the experiential learning perspective [11] to understand the mechanisms through which individuals' experiences with AI advice may assimilate them to AI and reinforce their acceptance of AI advice and choice adjustment behaviors.

\subsection{Individuals' assimilation to AI advice}

Individuals tend to learn with practice [16], and individuals' experiences with AI can provide opportunities to build knowledge about their decision making and to evaluate the accuracy of AI advice. Individuals' attitudes can evolve based on their interactions with technologies [6]. By accepting or rejecting AI's recommendations, individuals can evaluate their performance based on their experiences using AI. They perceive certain levels of performance by both rejecting and accepting AI advice. The experience may renew their knowledge about the decision contexts and AI. While doing so, individuals may learn about how AI makes judgments and whether they need to emulate the judgments. They can apply their learning to subsequent decision making. Considering that $\mathrm{AI}$ is believed to have higher accuracy than humans ${ }^{1}$, individuals' initial estimates may be similar to those of $\mathrm{AI}$ advice $^{2}$ as they have more experiences with AI-aided decision making. Hence, we suggest the following hypothesis:

Hypothesis 1: The difference between AI advice and individuals' initial estimates decreases as individuals gain AI-assisted decision-making experience.

\footnotetext{
${ }^{2}$ We provided AI advice to individuals after they made initial choices to more accurately identify the assimilation of users to AI.
} 


\subsection{Acceptance of and relative dependence on AI advice}

According to the anchoring-and-adjustment perspective $[7,17,18]$, individuals who are confident in their initial estimates tend to cease making adjustments and deliver these estimates. On the contrary individuals who do not have such confidence may feel the need to modify their estimates. This perspective assumes that individuals who are motivated to modify their initial estimates tend to generate estimates that are farther from the anchored estimates [18]. Clarifying this, Simmons et al. [15] suggest that corrections of initial estimates can occur both far from and close to initial estimates depending on the decision makers' confidence about the direction of adjustment from anchors. That is, when individuals are certain about the direction of adjustment but perceive that their adjustment is insufficient, they are more likely to adjust their initial estimates.

Applying this to our research context, if individuals believe that their initial estimates (based on their understanding of existing information) diverge from AI advice, they are more likely to motivate to change their initial estimates, moving them closer to those provided by AI. On the contrary, if individuals believe that their initial estimates are close to those given by AI, they may think that the estimates are reasonable and feel satisfied [19]. They are also less likely to feel the need to recalibrate their estimates based on AI advice, instead sustaining their initial estimates' proximity to the advice. In this decision-making context, AI advice may act as a standard of comparison [20,21]. In this way, similar to psychological theories of social proof [22], individuals can infer their final estimates from AI advice. Thus, they may have increased certainty about their initial judgments [23] and not feel the need to adjust their estimates when the AI advice is close to their initial estimates. This results in low acceptance of AI advice, followed by adjustment of initial estimates. Thus, we suggest the following hypothesis:

Hypothesis 2: Greater differences between AI advice and individuals' initial estimates lead to their greater acceptance of AI advice.

Acceptance of AI advice may not mean that individuals totally follow AI advice. If so, how much do individuals tend to change initial judgments after accepting AI advice as a reference? Researchers have generally observed that individuals focus more on anchor-consistent information; hence, selective accessibility tends to produce assimilative effects on individuals' judgments [13, 24]. Thus, individuals may keep their final choices closer to AI advice when the advice is similar to their initial estimates, because they may be more confident about the direction of their choice shifts. We suggest two mechanisms through which individuals are more likely to have confidence and exhibit the typical assimilative effects of anchors. First, individuals attempt to increase their cognitive efficiency by using mental models [25], which are representations of reality that allow individuals to make sense of the world in their conceptions of causality, expectations, and beliefs [26]. In other words, individuals may disregard information that should be considered if it does not correspond with their mental models. Such biased perceptions can make decision makers select information that supports their preexisting beliefs [18]. Several studies [e.g., 27] have shown that individuals tend to pay more (less) attention to (dis)confirmatory information in organizational decision-making contexts. Researchers have also found that similarities between a user and a recommendation agent are positively related to behavioral beliefs, particularly when users' decision making is more likely to be preferential and driven by heuristics [28, 29]. Second, individuals tend to exhibit egocentric biases, causing them to downplay the advice of other (more accurate) people or entities [30]. Accordingly, individuals are quite attached to and prefer to follow their intuitive judgments even while explicitly recognizing that other judgments are objectively correct [31]. Individuals are less likely to give higher weight to their own opinions [32] or ignore the advice that they receive [33]. The pursuit of cognitive efficiency and egocentric bias both can occur in anchoring contexts, creating a push-away effect, which leads to fewer estimates close to the advice [34].

Applying this reasoning to AI-aided decision making, even if $\mathrm{AI}$ is capable of making more accurate estimates than individuals, individuals may not be free from their own initial estimates due to their pursuit of cognitive efficiency and their egocentric biases. Though individuals can accumulate task-specific knowledge and adapt to AI-aided decision contexts, they may not be free from the anchoring bias, as evidenced by experts' susceptibility to anchoring [35]. For instance, Meub and Proeger [36] found that even after individuals accumulate task-specific knowledge based on comprehensive information about and repetition of identical tasks, they may still exhibit anchoring bias despite the modest learning effect. Hence, when the difference between an initial estimate and AI advice is greater, individuals will continue to select a final estimate that is relatively closer to the initial estimate, representing an assimilative effect [24]. We propose the concept of relative dependence on AI advice, representing the difference between an individual's final choice and their initial choice vis-à-vis the AI advice. This means that when individuals make final decisions closer to the AI advice than to their initial estimates, 
they are more likely to depend on the AI advice. We suggest the following hypothesis:

Hypothesis 3: Greater differences between AI advice and individuals' initial estimates lead to their less relative dependence on AI advice.

\subsection{Influence of multiple anchors}

Decision-making environments in organizations can have multiple anchors, which may determine the focal anchor's effect on decision making under uncertainty [37]. Although their initial estimates as an anchor may influence the role of AI advice, individuals are also exposed to other informational clues in their decision-making processes (e.g., experts' opinions or decisions made in similar situations). Prior studies have focused primarily on single-anchor contexts [9, 38]. Although few in number, prior studies have discovered some common mechanisms in the multiple-anchor context. For instance, though any anchor in multipleanchor contexts can exert some effects on final decisions [38, 39], the most highly applicable anchor has the strongest effect on the final decision [9].

Based on these findings, we posit that when multiple anchors (e.g., previous-year credit scores and initial estimates) are available, it may mitigate the assimilation of individuals' initial estimates to AI advice. This is because individuals possessing incomplete information are more likely to employ other information sources to increase their accuracy, when uncertainty is irreducible. Individuals may recognize the advantages of considering AI advice while they are adapting to decision-making contexts. In doing so, they can compare their initial estimates, a second anchor, and $\mathrm{AI}$ advice and then decide to depend on AI advice more selectively. In this decision contexts, decision makers may rely more on past decisions as a reference [37]. Hence, we propose the following hypothesis:

Hypothesis 4: When individuals are exposed to a second anchor, the difference between individuals' initial estimates and AI advice is less likely to decrease as they gain more AI-assisted decision-making experience, compared to when not exposed to a second anchor.

The presence of multiple anchors mitigates the effect of any single anchor [9, 37]. As individuals are less likely to rely primarily on AI advice when in the presence of a second anchor, the influence of the difference between AI advice and individuals' initial choice on their accuracy may decline as well. Because multiple anchors are available, individuals may not need to rely solely on the difference between their initial choice and AI advice in their decision makings. Instead, they may compare their initial estimates, AI advice, and a second anchor and then make their final decision. Put differently, even if individuals' initial estimates are different from AI advice, they may consider a second anchor's value. Particularly in the research context employing previous-year credit score as a second anchor, prior studies have demonstrated that individuals are more likely to pay attention to previous decisions made by others in comparable situations rather than maintaining an anchor [37], as past comparable estimates offer social proof [40]. Formally,

Hypothesis 5: When individuals are exposed to a second anchor, the positive effect of the difference between AI advice and their initial estimate on their acceptance of the advice is weaker, compared to when not exposed to a second anchor.

Hypothesis 6: When individuals are exposed to a second anchor, the negative effect of the difference between AI advice and their initial estimate on their relative dependence on AI advice is weaker, compared to when not exposed to a second anchor.

\subsection{Effect of AI advice on decision accuracy}

The accuracy of individuals' final estimates depends on their efforts to adjust their initial estimates. Thus, individuals' acceptance of AI advice alone may not enhance their accuracy. Given the uncertainty surrounding the true value being estimated, individuals have a range of estimates that they believe plausible. They are likely to cease making adjustments if they have arrived at a satisfactory (rather than the most accurate) estimate [19]. This "satisficing" often leads to egocentrically biased responses at the expense of enhanced accuracy [19] due to selective accessibility; in turn, this selective accessibility stems from individuals' efficiency pursuits while employing mental models [25]. Applying this rationale to this research context, individuals' acceptance of AI advice may imply that they have neither satisficed nor intend to finish their adjustments. Instead, they intend to reconsider the accuracy of their initial estimates. Thus, the accuracy of individuals' final estimates may increase through these effortful processes when accepting AI advice. However, we should note that individuals are not entirely free from egocentric biases, which can decrease their accuracy even when they intentionally adjust the initial estimates. Similarly, researchers have found that individuals tend to adjust approximately $30-35 \%$ of their estimates closer to advice received from others, which can hinder accuracy [42]. Even if individuals attempt to adjust their estimates, they tend to average their own judgment with the advice received [3], which can prevent them from reaping the full benefits of the external advice. Hence, we focus on the effect of relative dependence on the AI advice - that is, the difference between an individual's final choice and their initial choice vis-à-vis the $\mathrm{AI}$ 
advice - on decision accuracy. We posit that high levels of relative dependence on AI advice- rather than mere acceptance of AI advice -can represent the outcomes of individuals' debiasing efforts, which can yield higher decision accuracy. Formally,

Hypothesis 7: Relative dependence on AI advice increases the accuracy of individuals' final estimates.

\section{Method}

\subsection{Overview of experiments}

This study aimed to identify whether AI can help individuals overcome anchoring biases while also extending the research literature to multiple-anchor, AIaided decision-making contexts. Our goal was to explain how and when a potentially salient reference in decision making, AI advice, can influence individuals' dependence on AI advice and decision-making accuracy. We developed a 2 (similarity vs. nonsimilarity) X 2 (previous-year credit score vs. no previous-year credit score) between-subjects design. We conducted two longitudinal experiments using corporate credit ratings, where machine learning techniques and related AI systems are widely developed and utilized throughout organizations [43]. Experiment 1 aimed to determine whether individuals are more likely to take advice when their initial estimates are closer to that advice in the two-stage anchoring process (the independent initial estimate-then-revise-estimate sequence)-adopting the advice, then reflecting and shifting to a self-generated value. Experiment 2 explored whether the results found in Experiment 1 could be replicated in multiple-anchor contexts, which characterize many task environments and affect anchoring's ability to influence judgments under uncertain conditions [9, 34].

We recruited participants from major universities in South Korea, providing 15,000 Korean won (KRW, 1 $\mathrm{USD}=1,100 \mathrm{KRW}$ in January, 2021) for their participation. We also motivated them to enhance their learning and decision accuracy efforts by offering them 50,000 to $100,000 \mathrm{KRW}$ based on their performance. We distributed online links to individuals via email, which indicated their voluntary participation in the experiments. Participants had to learn how to rate credit scores based on company-relevant information before entering the experiment. We emphasized that the AI advice that they were receiving would not be $100 \%$ accurate and thus that they needed to employ the advice strategically. Participants were required to complete three experimental sessions. In each session, they completed 10 corporate credit rating tasks over approximately 10 days. All participants encountered the same questions but in different orders. Once participants had finished a session, they could move on to the next session after five days. In total, they assigned credit ratings to 30 companies for three sessions over approximately two months. We anonymized the names of firms, meaning that participants could not rate their credit based on external sources.

Participants estimated each company's credit at two timepoints: before and after receiving AI advice. Thus, participants made initial estimates based purely on information provided about a company (Experiment 1) or this information combined with the company's previous-year credit score (Experiment 2). Then, participants received AI advice. After receiving this advice, participants had the opportunity to adjust their initial estimates only when they answered "Yes" to the question "Will you accept AI advice and change the initial estimate?" That is, participants estimated an initial value and adjusted it as needed to arrive at a final judgment. After completing one session, participants viewed their aggregated performance per session. That is, they did not know the accuracy of decision makings during the sessions.

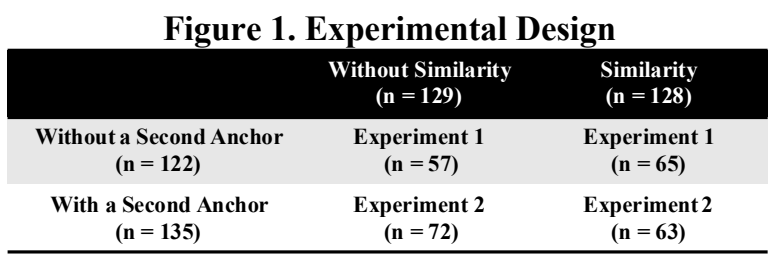

\subsection{AI-based recommendation system development}

We developed AI-based systems to manipulate the conditions: (1) one system recommending credit scores based only on historical data for credit events, with the other providing credit scores based on both historical data and users' initial estimates; and (2) one system providing companies' previous-year credit scores before participants estimated an initial credit rating, with the other not offering this information. We embedded machine learning algorithms in the systems. We employed the caret and xgboost packages as well as the linear regression, random forest, and gradientboosted decision tree techniques offered by R statistics. We used credit events obtained from Korea Enterprise Data, which is one of the largest companies providing corporate credit information in South Korea for banks and government entities. Within the database, credit scores were classified into 20 rating categories: 1 (highest) $\sim 20$ (cannot be graded)). The initial dataset included more than 113,000 credit events from 2002 to 2017, while the final set included 842 year-company paired credit events from 2015 to 2017, as some data (e.g., intellectual properties [IPs]) had a much shorter history available and many companies do not survive 
longer than three years. For AI training purposes, we included 18 financial (e.g., total assets, paid-in capital, annual sales) and non-financial attributes (e.g., number of international IPs and company locations) that have been identified by prior studies [e.g., 44] as influencing corporate credit ratings. We also provided these attributes to the participants. Both the AI systems and the participants employed the same information to estimate credit scores. After training the AI with the dataset, we compared AI-recommended credit ratings with ones taken from the database. Without learning users' heuristics, AI advice had a root mean squared error (RMSE) of 2.513. That is, when the AI system recommended 15, the true value would range from 12.487 to 17.513 with $99 \%$ probability.

Participants. We recruited MBA students and senior-level students in several major business schools in South Korea, who had varied experiences and backgrounds. None of them participated more than once in any individual experiment. In Experiment 1 and 2, 122 and 135 participants completed the three sessions.

\subsection{Measures}

Acceptance of AI Advice. For $i^{\text {th }}$ individual on the $q^{\text {th }}$ decision task, we measured individuals' acceptance of AI advice (Accept iq $_{\text {) }}$ with the question "Will you accept AI advice and change the initial estimate?" (Yes $=1$, No $=0$ ).

Relative Dependence on AI Advice over Initial Estimates. We employed the Judge Advisor System (JAS) paradigm [45], to measure relative dependence on AI advice over initial estimates $\left(D e p A I_{i q}\right)$ by calculating $\frac{\left|F E_{i q}-I E_{i q}\right|}{\left(\left|F E_{i q}-A I A_{i q}\right|+\left|F E_{i q}-I E_{i q}\right|\right)}$, where $F E_{i q}$ is the final estimate, $I E_{i q}$ is the initial estimate, and $A I A_{i q}$ is the value of $\mathrm{AI}$ advice. We should note that when $F E_{i q}$ equaled $I E_{i q}$ or $A I A_{i q}$, we assigned difference scores of $0.01 . D e p A I_{\text {iq }}$ had a value ranging from 0 (abandoning the AI advice) to 1 (abandoning the initial estimate).

Decision Accuracy. We measured the accuracy (Accuracy iq $_{\text {) }}$ of individuals' decisions by comparing their final estimates with the values in the database as 20 - $\mid F E_{i q}$-Database Valueiq $\mid$.

Experience. For the $i^{\text {th }}$ individual on the $q^{\text {th }}$ decision task, we operationalized participants' experience by counting the number of credit scoring decision tasks.

Difference between AI Advice and Initial Estimates. We computed the difference between the initial estimate and the $\mathrm{AI}$ advice $\left(D i f_{i q}\right)$ as $\left|I E_{i q}-A I A_{i q}\right|$.

Control Variables. We included several pretreatment control variables to reduce residual variation and increase our estimates' precision. We included educational background, work experience, and current occupation to control for the effect of domain knowledge on the use of AI advice [1]. We also included participants' age, gender, and university to control for unobserved effects on the dependent variables and for the anchoring effect [3]. Finally, we included session dummy variables to control for learning effects across sessions and other unobserved effects within sessions.

\subsection{Analyses}

We built a dynamic panel dataset based on observations of the participants' decisions and behaviors to estimate the parameters. We measured the variables repeatedly, and they had a nested naturedecision tasks nested in sessions, nested in individuals. The individual and session-specific components should be correlated with the regressors $(\mathrm{E}[\mathrm{X}, \psi] \neq 0$ and/or $\mathrm{E}[\mathrm{X}, \xi] \neq 0)$. That is, the common error component over individuals and sessions may induce serial correlations across the composite error terms [46]. Durbin-Watson tests failed to reject the null hypothesis that serial correlations were equal to zero for the variables $(p=$ 1.000 for Dif $_{i q}, p=.733$ for Accept $_{i q}, p=.168$ for $D_{e p A I}$, and $p=.568$ for Accuracy $_{i q}$ ). Thus, we conducted generalized linear modeling (GLM) with a general feasible generalized least square (FGLS) method.

\section{Results}

The results of Hausman test revealed significant differences between the fixed-effect and random-effects models when we employed dependent variables. Thus, we employed the fixed-effects model. As shown in Model 1-1 in Table 1, Difiq which represents the opposite of assimilation, increased as individuals assigned more credit ratings (coefficient $=0.0369, p$ $<.001$ ); thus, H1 was not supported. In Model 1-1, the effect of experience on $D i f_{i q}$ depended on the existence of a second anchor (coefficient $=-0.0322, p<.001$ ). We split the sample into two groups - those not exposed to a second anchor (Experiment 1) and those exposed to a second anchor (Experiment 2). As shown in Model 1-2, as participants assigned more credit ratings without a second anchor, Dif $f_{i q}$ decreased (coefficient $=-0.0392, p$ $<.001$ ). On the contrary, as shown in Model 1-3, as individuals completed more decision tasks with a second anchor, Difiq increased (coefficient $=0.0820, p$ $<.01)$. These two coefficients were statistically different $(t=13.719, p<.01)$. Overall, without extra information that can influence individuals' initial estimates, users are more likely to assimilate to AI advice over time. However, a second anchor may reduce assimilation. These results support $\mathrm{H} 4$. 
Table 1. Results: User-AI Difference

\begin{tabular}{|c|c|c|c|c|c|c|}
\hline \multirow[b]{3}{*}{ Dependent Variable: User-AI Difference } & \multirow{2}{*}{\multicolumn{2}{|c|}{$\begin{array}{c}\text { Experiments } 1+2 \\
(\mathrm{n}=7710)\end{array}$}} & \multirow{2}{*}{\multicolumn{2}{|c|}{$\begin{array}{c}\begin{array}{c}\text { Experiment } 1 \\
(\mathrm{n}=3660)\end{array} \\
\text { Model } 1-2\end{array}$}} & \multirow{2}{*}{\multicolumn{2}{|c|}{$\begin{array}{c}\begin{array}{c}\text { Experiment } 2 \\
(\mathrm{n}=4050)\end{array} \\
\text { Model } 1-3\end{array}$}} \\
\hline & & & & & & \\
\hline & Coef & Error & Coef & Error & Coef & Error \\
\hline D1_Session & $-1.5510^{* * *}$ & 0.0720 & $-0.6283^{* * *}$ & 0.0975 & $-2.6063^{* * *}$ & 0.0662 \\
\hline D2_Session & $-1.9284^{* * *}$ & 0.1364 & -0.2896 & 0.1807 & $-3.6390^{* * *}$ & 0.1283 \\
\hline Experience & $0.0369^{* * *}$ & 0.0072 & $-0.0392^{* * *}$ & 0.0097 & $0.0820^{* * *}$ & 0.0062 \\
\hline D_Second Anchor X Experience & $-0.0322^{* * *}$ & 0.0029 & & & & \\
\hline Individual and Demographic Dummies & Yes & & Yes & & Yes & \\
\hline Multiple R-squared & \multicolumn{2}{|c|}{0.185} & \multicolumn{2}{|l|}{0.157} & \multicolumn{2}{|c|}{0.222} \\
\hline
\end{tabular}

As shown in Models 2-1 and 2-2 in Table 2, after controlling for the effect of experience, $D i f_{i q}$ positively influenced Accept iq $_{\text {(coefficient }}=0.0655, p<.001$ and $0.0748, p<.001$ ), supporting H2. Additionally, the interaction between Difiq and D_Second Anchor negatively affected Accept $_{i q}$ (coefficient $=-0.0188, p$ $<.001$ ). Thus, the effect of Difiq on Accept $_{i q}$ depends on the existence of a second anchor. We split the sample into two groups and conducted subgroup analysis. As shown in Models 2-3 and 2-4, Dif positively influenced Acceptiq more strongly in the absence of a second anchor (coefficient $=0.0705, p$ $<.001$ ) compared to when a second anchor was present $($ coefficient $=0.0496, p<.001)$. The difference between the two groups' coefficients was statistically significant $(t=8.731, p<.05)$. This indicates that when individuals were exposed to a second anchor, the positive effect of Difiq on Acceptiq was weaker, supporting H5.

Table 2. Results: Acceptance of AI Advice

\begin{tabular}{|c|c|c|c|c|c|c|c|c|}
\hline \multirow[b]{3}{*}{ Dependent Variable: AI Use } & \multicolumn{4}{|c|}{$\begin{array}{c}\text { Experiments } 1+2 \\
(\mathrm{n}=7710)\end{array}$} & \multirow{2}{*}{\multicolumn{2}{|c|}{$\begin{array}{c}\text { Experiment } 1 \\
(n=3660)\end{array}$}} & \multirow{2}{*}{\multicolumn{2}{|c|}{$\begin{array}{c}\begin{array}{c}\text { Experiment } 2 \\
(\mathrm{n}=4050)\end{array} \\
\text { Model } 2-4\end{array}$}} \\
\hline & \multicolumn{2}{|c|}{ Model 2-1 } & \multicolumn{2}{|c|}{ Model 2-2 } & & & & \\
\hline & Coef & Error & Coef & Error & Coef & Error & Coef & Error \\
\hline$\overline{\text { D1_Session }}$ & $0.1085^{* * *}$ & 0.0132 & $0.0966^{* * *}$ & 0.0134 & $0.0738^{* * *}$ & 0.0179 & $0.0369^{* * *}$ & 0.0136 \\
\hline $\mathrm{D} 2$ Session & $0.2073^{* * *}$ & 0.0279 & $0.1884^{* * *}$ & 0.0281 & $0.1655^{* * *}$ & 0.0405 & $0.1257^{* * *}$ & 0.0342 \\
\hline Experience & $-0.0056^{* * *}$ & 0.0014 & $-0.0064^{* * *}$ & 0.0016 & $-0.0064^{* *}$ & 0.0020 & -0.0015 & 0.0017 \\
\hline User-AI Difference (Dif) & $0.0655^{* * *}$ & 0.0017 & $0.0748^{* * *}$ & 0.0024 & $0.0705^{* * *}$ & 0.0019 & $0.0496^{* * *}$ & 0.0019 \\
\hline D_Second Anchor X Dif & & & $-0.0188^{* * *}$ & 0.0034 & & & & \\
\hline D Second Anchor X Experience & & & $0.0030^{*}$ & 0.0013 & & & & \\
\hline Individual and Demographic Dummies & \multicolumn{2}{|c|}{ Yes } & \multicolumn{2}{|c|}{ Yes } & \multicolumn{2}{|c|}{ Yes } & \multicolumn{2}{|l|}{ Yes } \\
\hline Multiple R-squared & \multicolumn{2}{|c|}{0.291} & \multicolumn{2}{|c|}{0.311} & \multicolumn{2}{|c|}{0.353} & \multicolumn{2}{|c|}{0.222} \\
\hline
\end{tabular}

We included only observations who accepted AI advice in this analysis in our analysis on the relative dependence on AI. As shown in Models 3-1 and 3-2 (Table 3), after controlling for the effect of experience, $D i f_{i q}$ negatively influenced DepAI $I_{i q}$ (coefficient = $0.0235, p<.001$ and $-0.0134, p<.001$ ), supporting H3. $D i f_{i q}$ negatively influenced $D e p A I_{i q}$ more strongly in the presence of a second anchor (coefficient $=-0.0138, p$ $<.001$ ), compared to when a second anchor was absent (coefficient $=-0.0022, p<.001)$. The difference between the two groups' coefficients was statistically significant $(t=21.789, p<.05)$. Thus, H6 was not supported.

We expected that $D e p A I_{i q}$ would increase decision accuracy. As shown in Models 4-2, 4-3, and 4-4 in Table 4, after controlling for the effects of experience, DepAI positively influenced accuracy, with coefficients of $1.1260(p<.001), 3.2278(p<.001)$, and $2.1539(p$ $<.001$ ), respectively. The effect is consistently positive, independent of a second anchor's existence. Thus, H7 was supported.

\section{Discussion and Conclusions}

AI advice's influence on individuals' judgments and decision making has been garnering much attention among researchers, but there is still limited theoretical understanding in this regard. In the context of two-stage anchoring processes (the independent estimate-thenrevise-estimate sequence), which reflects organizational decision-making situations [34], the results clearly demonstrate that $\mathrm{AI}$ advice may not fully eliminate individuals' anchoring biases (e.g., [13, 14], [23]) even when learning occurs in repeated tasks [36]. 
Furthermore, the difference between individuals' initial estimates and AI advice can increase effortful adjustments but can also paradoxically motivate individuals to stay close to their initial estimates. That is, the results support our expectations that while adjusting their estimates according to anchors, individuals tend to exhibit egocentric biases in AI-aided decision-making contexts. This may be a reason why individuals are often unable to reap the full benefits of AI advice and why accuracy may not sufficiently increase as expected.

Table 3. Results: Dependence on AI Advice Relative to Individuals' Initial Estimate

\begin{tabular}{|c|c|c|c|c|c|c|c|c|}
\hline \multirow{3}{*}{$\begin{array}{l}\text { Dependent Variable: Relative } \\
\text { Dependence on AI Advice } \\
\text { (only for the observations } \\
\text { accepting AI advice) }\end{array}$} & \multicolumn{4}{|c|}{$\begin{array}{c}\text { Experiments } 1+2 \\
(n=2520)\end{array}$} & \multirow{2}{*}{\multicolumn{2}{|c|}{$\begin{array}{c}\begin{array}{c}\text { Experiment } 1 \\
(\mathrm{n}=1308)\end{array} \\
\text { Model 3-3 } \\
\end{array}$}} & \multirow{2}{*}{\multicolumn{2}{|c|}{$\begin{array}{c}\begin{array}{c}\text { Experiment } 2 \\
(\mathrm{n}=1212)\end{array} \\
\text { Model 3-4 }\end{array}$}} \\
\hline & \multicolumn{2}{|c|}{ Model 3-1 } & \multicolumn{2}{|c|}{ Model 3-2 } & & & & \\
\hline & Coef & Error & Coef & Error & Coef & Error & Coef & Error \\
\hline D1_Session & $-0.0945^{* * *}$ & 0.0039 & $-0.0757^{* * *}$ & 0.0049 & -0.0044 & 0.0070 & $0.0811^{* * *}$ & 0.0058 \\
\hline D2 2 Session & $-0.1591^{* * *}$ & 0.0065 & $-0.2689^{* * *}$ & 0.0071 & $0.0357^{* * *}$ & 0.0086 & $0.1307^{* * *}$ & 0.0106 \\
\hline Experience & $0.0041^{* * *}$ & 0.0003 & $0.0138^{* * *}$ & 0.0003 & $0.0016^{* * *}$ & 0.0003 & $-0.0057^{* * *}$ & 0.0004 \\
\hline User-AI Difference (Dif) & $-0.0235^{* * *}$ & 0.0006 & $-0.0134^{* * *}$ & 0.0006 & $-0.0022^{* * *}$ & 0.0005 & $-0.0138^{* * *}$ & 0.0005 \\
\hline D_Second Anchor X Dif & & & $-0.0575^{* * *}$ & 0.0010 & & & & \\
\hline D_Second Anchor X Experience & & & $-0.0052^{* * *}$ & 0.0004 & & & & \\
\hline Individual and Demographic Dummies & \multicolumn{2}{|c|}{ Yes } & \multicolumn{2}{|c|}{ Yes } & \multicolumn{2}{|c|}{ Yes } & \multicolumn{2}{|c|}{ Yes } \\
\hline Multiple R-squared & \multicolumn{2}{|c|}{0.658} & \multicolumn{2}{|c|}{0.649} & 0.676 & & \multicolumn{2}{|l|}{0.669} \\
\hline
\end{tabular}

Table 4. Results: Decision Making Accuracy

\begin{tabular}{|c|c|c|c|c|c|c|c|c|}
\hline \multirow[b]{3}{*}{ Dependent Variable: Accuracy } & \multicolumn{4}{|c|}{$\begin{array}{c}\text { Experiments } 1+2 \\
(\mathrm{n}=7710)\end{array}$} & \multirow{2}{*}{\multicolumn{2}{|c|}{$\begin{array}{c}\begin{array}{c}\text { Experiment } 1 \\
(\mathrm{n}=3660)\end{array} \\
\text { Model } 4-3 \\
\end{array}$}} & \multirow{2}{*}{\multicolumn{2}{|c|}{$\begin{array}{c}\begin{array}{c}\text { Experiment } 2 \\
(\mathrm{n}=4050)\end{array} \\
\text { Model 4-4 }\end{array}$}} \\
\hline & \multicolumn{2}{|c|}{ Model 4-1 } & \multicolumn{2}{|c|}{ Model 4-2 } & & & & \\
\hline & Coef & Error & Coef & Error & Coef & Error & Coef & Error \\
\hline D1_Session & $1.3274^{* * *}$ & 0.0449 & $1.8118^{* * *}$ & 0.0435 & $1.3094^{* * *}$ & 0.0382 & $1.1075^{* * *}$ & 0.0327 \\
\hline D2_Session & $1.7697^{* * *}$ & 0.1059 & $1.8624^{* * *}$ & 0.0838 & $1.8483^{* * *}$ & 0.0674 & $1.2177^{* * *}$ & 0.0681 \\
\hline Experience & $-0.0276^{* * *}$ & 0.0053 & 0.0012 & 0.0047 & $-0.0302^{* * *}$ & 0.0026 & $0.0150^{* * *}$ & 0.0027 \\
\hline \multicolumn{9}{|c|}{ D_Acceptance of AI Advice (D_AAI, Dummy) } \\
\hline Relative Dependence on AI Advice (RD) & & & $1.1260^{* * *}$ & 0.0757 & $3.2278^{* * *}$ & 0.0552 & $2.1539^{* * *}$ & 0.0357 \\
\hline D_Second Anchor X Experience & & & $-0.0275^{* * *}$ & 0.0024 & & & & \\
\hline \multicolumn{9}{|l|}{ D_Second Anchor X D_AAI } \\
\hline D_Second Anchor X R $\bar{D}$ & & & $0.6619^{* * *}$ & 0.0822 & & & & \\
\hline Individual and Demographic Dummies & \multicolumn{2}{|c|}{ Yes } & \multicolumn{2}{|c|}{ Yes } & \multicolumn{2}{|l|}{ Yes } & \multicolumn{2}{|c|}{ Yes } \\
\hline Multiple R-squared & \multicolumn{2}{|c|}{0.218} & \multicolumn{2}{|c|}{0.314} & \multicolumn{2}{|l|}{0.296} & \multicolumn{2}{|c|}{0.333} \\
\hline
\end{tabular}

$\dagger \mathrm{p}<.10, * \mathrm{p}<.05, * * \mathrm{p}<.01, * * * \mathrm{p}<.001$

Several studies (e.g., [28], [29]) have demonstrated that individuals are more likely to accept algorithmic recommendations similar to their own choices in heuristic decision-making contexts. However, the present study shows that individuals can be still biased toward their initial judgments even after accepting algorithmic advice; thus, mere acceptance of AI advice may not be sufficient to shift choices. Hence, our research extends past findings by showing that the impact of AI advice may differ in the two stages of decision making: (1) intentions to accept AI advice and (2) adjustments to initial estimates. Further, the results indicate that multiple anchors have potential to increase the negative influence of the difference between self-generated anchors (initial estimates in this study) and AI advice on the dependence on AI advice. However, when AI advice is similar to the value of a second anchor (but not an initial estimate) it can increase the dependence on the advice, helping individuals debias themselves from their anchors. Considering that few studies have examined multiple anchors [9], this study expands our understanding of second anchors' impact on AI-aided decision making by determining the roles of its consistency with other information sources.

Our study's results suggest that AI advice may lower decision accuracy in contexts where individuals do not sufficiently change their initial judgments. Further, individuals in the presence of a second anchor may exhibit decreasing decision accuracy by accepting AI advice. However, individuals' decision accuracy improves if they not only accept AI advice but also maintain proximity to the advice. Hence, this study demonstrates whether and how AI can enhance decision accuracy, and it contributes to our theoretical understanding of the relationship between AI use and decision performance. 
Investment in AI-based DSS has increased in recent years across industries, with the hope of enhancing decision-making effectiveness But, this study indicates that individuals may not reap the full benefits of AI-based DSS without proper decision strategies that account for biases and learning. Thus, our research shows that when implementing AI technologies, organizations must also train their employees in leveraging AI advice optimally. Such training should focus on individuals' paradoxical choice shifts. Namely, if AI advice is statistically more accurate than their judgments, employees must be informed that their final decisions may be enhanced by more closely following the AI advice.

\section{References}

[1] P. Choudhury, E. Starr, and R. Agarwal, "Machine learning and human capital complementarities: Experimental evidence on bias mitigation," Strategic Management Journal, vol. 41, no. 8, pp. 1381-1411, 2020.

[2] B. Cowgill and C. E. Tucker, "Algorithmic fairness and economics," The Journal of Economic Perspectives, 2020.

[3] J. M. Logg, J. A. Minson, and D. A. Moore, "Algorithm appreciation: People prefer algorithmic to human judgment," Organizational Behavior and Human Decision Processes, vol. 151, pp. 90-103, 2019.

[4] B. J. Dietvorst and S. Bharti, "People reject algorithms in uncertain decision domains because they have diminishing sensitivity to forecasting error," Psychological science, vol. 31, no. 10, pp. 1302-1314, 2020.

[5] B. J. Dietvorst, J. P. Simmons, and C. Massey, "Overcoming algorithm aversion: People will use imperfect algorithms if they can (even slightly) modify them," Management Science, vol. 64, no. 3, pp. 1155-1170, 2018.

[6] W. J. Orlikowski, "The sociomateriality of organisational life: considering technology in management research," Cambridge journal of economics, vol. 34, no. 1, pp. 125-141, 2010.

[7] A. Tversky and D. Kahneman, "Judgment under uncertainty: Heuristics and biases," science, vol. 185, no. 4157, pp. 1124-1131, 1974.

[8] G. Adomavicius, J. C. Bockstedt, S. P. Curley, and J. Zhang, "Do recommender systems manipulate consumer preferences? A study of anchoring effects," Information Systems Research, vol. 24, no. 4, pp. 956-975, 2013.

[9] G. Whyte and J. K. Sebenius, "The effect of multiple anchors on anchoring in individual and group judgment," Organizational behavior and
We should note that this study did not measure the psychological constructs and identify their influence on choice shifts, as it focused on data directly reflecting initial and final choices. Such objective measures have their benefits, but not assessing the psychological mechanisms underlying the choice shifts does represent a limitation. Future research could assess these relevant constructs, for instance, by measuring the confidence that individuals have in their initial judgments and examining how the perceived quality of the AI advice influences individuals' behaviors. Thus, future studies could build on our speculations and methodology by focusing on the underlying mechanisms of human behaviors in AIaided decision-making contexts.

human decision processes, vol. 69, no. 1, pp. 7585, 1997.

[10] D. Kahneman, Thinking, fast and slow. Macmillan, 2011.

[11] D. A. Kolb, Experiential learning: Experience as the source of learning and development. FT press, 2014.

[12] D. Arnott and S. Gao, "Behavioral economics for decision support systems researchers," Decision Support Systems, vol. 122, p. 113063, 2019.

[13] L. Rhue, "Racial influence on automated perceptions of emotions," Available at SSRN 3281765, 2018.

[14] L. Rhue, "Anchored to Bias: How AI-Human Scoring Can Induce and Reduce Bias Due to the Anchoring Effect," Available at SSRN 3492129, 2019.

[15] J. P. Simmons, R. A. LeBoeuf, and L. D. Nelson, "The effect of accuracy motivation on anchoring and adjustment: Do people adjust from provided anchors?," Journal of personality and social psychology, vol. 99, no. 6, p. 917, 2010.

[16] X. N. Deng and L. Chi, "Knowledge boundary spanning and productivity in information systems support community," Decision Support Systems, vol. 80, pp. 14-26, 2015.

[17] N. Epley and T. Gilovich, "Putting adjustment back in the anchoring and adjustment heuristic: Differential processing of self-generated and experimenter-provided anchors," Psychological science, vol. 12, no. 5, pp. 391-396, 2001.

[18] N. Epley and T. Gilovich, "The anchoring-andadjustment heuristic: Why the adjustments are insufficient," Psychological science, vol. 17, no. 4, pp. 311-318, 2006.

[19] N. Epley, B. Keysar, L. Van Boven, and T. Gilovich, "Perspective taking as egocentric anchoring and adjustment," Journal of personality and social psychology, vol. 87, no. 3, p. 327, 2004.

[20] G. Ku, A. D. Galinsky, and J. K. Murnighan, "Starting low but ending high: A reversal of the 
anchoring effect in auctions," Journal of Personality and social Psychology, vol. 90, no. 6, p. 975, 2006.

[21] T. Mussweiler, F. Strack, and T. Pfeiffer, "Overcoming the inevitable anchoring effect: Considering the opposite compensates for selective accessibility," Personality and Social Psychology Bulletin, vol. 26, no. 9, pp. 1142-1150, 2000.

[22] R. B. Cialdini, "The psychology of persuasion," New York, 1993.

[23] H. J. Wilson and P. R. Daugherty, "Collaborative intelligence: humans and AI are joining forces," Harvard Business Review, vol. 96, no. 4, pp. 114$123,2018$.

[24] G. B. Chapman and E. J. Johnson, "Anchoring, activation, and the construction of values," Organizational behavior and human decision processes, vol. 79, no. 2, pp. 115-153, 1999.

[25] H.-H. Huang, J. S.-C. Hsu, and C.-Y. Ku, "Understanding the role of computer-mediated counter-argument in countering confirmation bias," Decision Support Systems, vol. 53, no. 3, pp. 438-447, 2012.

[26] J. K. Doyle and D. N. Ford, "Mental models concepts for system dynamics research," System dynamics review: the journal of the System Dynamics Society, vol. 14, no. 1, pp. 3-29, 1998.

[27] D. Arnott, "Cognitive biases and decision support systems development: a design science approach," Information Systems Journal, vol. 16, no. 1, pp. 55-78, 2006.

[28] S. Al-Natour and I. Benbasat, "The adoption and use of IT artifacts: A new interaction-centric model for the study of user-artifact relationships," Journal of the Association for Information Systems, vol. 10, no. 9, p. 2, 2009.

[29] B. Xiao and I. Benbasat, "E-commerce product recommendation agents: Use, characteristics, and impact," MIS quarterly, pp. 137-209, 2007.

[30] I. Yaniv and E. Kleinberger, "Advice taking in decision making: Egocentric discounting and reputation formation," Organizational behavior and human decision processes, vol. 83, no. 2, pp. 260-281, 2000.

[31] K. Woolley and J. L. Risen, "Closing your eyes to follow your heart: Avoiding information to protect a strong intuitive preference," Journal of personality and social psychology, vol. 114, no. 2, p. 230, 2018.

[32] A. E. Mannes, "Are we wise about the wisdom of crowds? The use of group judgments in belief revision," Management Science, vol. 55, no. 8, pp. 1267-1279, 2009.

[33] J. B. Soll and R. P. Larrick, "Strategies for revising judgment: How (and how well) people use others' opinions," Journal of experimental psychology: Learning, memory, and cognition, vol. 35, no. 3, p. 780, 2009.

[34] C. A. Rader, J. B. Soll, and R. P. Larrick, "Pushing away from representative advice: Advice taking, anchoring, and adjustment," Organizational Behavior and Human Decision Processes, vol. 130, pp. 26-43, 2015.

[35] A. Furnham and H. C. Boo, "A literature review of the anchoring effect," The journal of socioeconomics, vol. 40, no. 1, pp. 35-42, 2011.

[36] L. Meub and T. E. Proeger, "Anchoring in social context," Journal of Behavioral and Experimental Economics, vol. 55, pp. 29-39, 2015.

[37] S. Malhotra, P. Zhu, and T. H. Reus, "Anchoring on the acquisition premium decisions of others," Strategic Management Journal, vol. 36, no. 12, pp. 1866-1876, 2015.

[38] N. Bhatia and B. C. Gunia, "'I was going to offer $\$ 10,000$ but...": The effects of phantom anchors in negotiation," Organizational Behavior and Human Decision Processes, vol. 148, pp. 70-86, 2018.

[39] M. Schaerer, D. D. Loschelder, and R. I. Swaab, "Bargaining zone distortion in negotiations: The elusive power of multiple alternatives," Organizational Behavior and Human Decision Processes, vol. 137, pp. 156-171, 2016.

[40] H. Rao, H. R. Greve, and G. F. Davis, "Fool's gold: Social proof in the initiation and abandonment of coverage by Wall Street analysts," Administrative Science Quarterly, vol. 46, no. 3, pp. 502-526, 2001.

[41] A.-S. Chaxel, "The impact of procedural priming of selective accessibility on self-generated and experimenter-provided anchors," Journal of Experimental Social Psychology, vol. 50, pp. $45-$ 51, 2014.

[42] V. Liberman, J. A. Minson, C. J. Bryan, and L. Ross, "Naïve realism and capturing the "wisdom of dyads"," Journal of Experimental Social Psychology, vol. 48, no. 2, pp. 507-512, 2012.

[43] M. Wang and $\mathrm{H}$. Ku, "Utilizing historical data for corporate credit rating assessment," Expert Systems with Applications, vol. 165, p. 113925 , 2021.

[44] Z. Huang, H. Chen, C.-J. Hsu, W.-H. Chen, and S. $\mathrm{Wu}$, "Credit rating analysis with support vector machines and neural networks: a market comparative study," Decision support systems, vol. 37, no. 4, pp. 543-558, 2004.

[45] J. A. Sniezek and T. Buckley, "Cueing and Cognitive Conflict in Judge-Advisor Decision Making," Organizational behavior and human decision processes, vol. 62, no. 2, pp. 159-174, 1995, doi: 10.1006/obhd.1995.1040.

[46] Y. Croissant and G. Millo, "Panel data econometrics in R: The plm package," Journal of statistical software, vol. 27, no. 2, 2008. 